\title{
A method for the expression of fibroblast growth factor 14 and assessment of its neuroprotective effect in an Alzheimer's disease model
}

\author{
Lusheng Wang ${ }^{1,2 \#}$, Rongrong Jing ${ }^{2 \#}$, Xing Wang ${ }^{2}$, Baohui Wang ${ }^{2}$, Keke Guo ${ }^{2}$, Jungang Zhao ${ }^{2}$, Shuang Gao $^{2}$, \\ Nuo $\mathrm{Xu}^{2}$, Xuan Xuan ${ }^{1}$ \\ ${ }^{1}$ Department of Dermatology, The First Affiliated Hospital of Wenzhou Medical University, Wenzhou, China; ${ }^{2}$ College of Life and Environmental \\ Sciences, Wenzhou University, Wenzhou, China \\ Contributions: (I) Conception and design: N Xu, X Xuan; (II) Administrative support: L Wang, R Jing; (III) Provision of study materials or patients: \\ X Wang, B Wang; (IV) Collection and assembly of data: K Guo, J Zhao; (V) Data analysis and interpretation: S Gao; (VI) Manuscript writing: All \\ authors; (VII) Final approval of manuscript: All authors. \\ \#These authors contributed equally to this work. \\ Correspondence to: Nuo Xu. College of Life and Environmental Sciences, Wenzhou University, Wenzhou, China. Email: seer.sino@hotmail.com; Xuan Xuan. \\ Department of Dermatology, The First Affiliated Hospital of Wenzhou Medical University, Wenzhou, China. Email: XuanXuanWY@hotmail.com.
}

Background: Fibroblast growth factor (FGF) 14 is a member of the FGF family that is mainly expressed in the central nervous system. FGF14 has a close association with the occurrence of neurodegenerative conditions; however, its significance in Alzheimer's disease (AD) has yet to be evaluated. Therefore, we sought to obtain a large amount of exogenous FGF14 protein and explore its effect in a cellular model of AD.

Methods: FGF14 protein was expressed in an Escherichia coli system using gene recombination technology. Purified protein was obtained through washing and renaturation of inclusion bodies combined with nickel column affinity chromatography. The AD model was established via Aß25-35-induced injury in PC12 cells. Changes in the levels of lactate dehydrogenase and malondialdehyde were detected, and the neuroprotective effect of recombinant human FGF14 (rhFGF14) was evaluated through double-fluorescence staining and flow cytometry apoptosis detection. For further exploration of rhFGF14-mediated regulation of mitogenactivated protein kinase (MAPK) signaling, western blot was employed.

Results: We successfully induced large amounts of insoluble rhFGF14. Following solubilization and refolding of the rhFGF14 from inclusion bodies, high purity rhFGF14 was purified by Nickel affinity column chromatography. The results showed that rhFGF14 alleviated A 325 -3-induced PC12 cell injury by inhibiting the phosphorylation of $\mathrm{p} 38$, extracellular signal-regulated kinase $1 / 2$, and c-Jun N-terminal kinase, thus suppressing the MAPK signaling pathway.

Conclusions: FGF14 performed a neuroprotective role in our in vitro AD model via its inhibition of MAPK signaling, highlighting its potential as a therapeutic drug for neurodegenerative conditions.

Keywords: Fibroblast growth factor 14 (FGF14); Alzheimer's disease (AD); protein expression; $\beta$-amyloid protein; neuroprotection

Submitted Apr 29, 2021. Accepted for publication Jun 11, 2021.

doi: 10.21037/atm-21-2492

View this article at: https://dx.doi.org/10.21037/atm-21-2492 


\section{Introduction}

Fibroblast growth factor (FGF) 14 is a biologically active protein found in the brain and pituitary gland, which promotes fibroblast growth and participates in embryonic development, angiogenesis, tissue repair, and other processes (1). As a member of the FGF homologous factor family, FGF14 is expressed in developing and mature nervous systems $(1,2)$, in which it maintains normal nervous system function via its regulation of neuronal plasticity and excitability. Research has discovered that mutations in the FGF14 gene are responsible for the neurodegenerative condition spinocerebellar ataxia (SAC27) (3), and that FGF14 deficiency impairs the maturation of hippocampal dentate gyrus cells, which may result in schizophrenia $(4,5)$.

Alzheimer's disease (AD) is a frequently occurring neurodegenerative disease in older people and is the key cause of dementia symptoms in patients over the age of 60 (6). $\mathrm{AD}$ is characterized by 3 major pathological signs: dysfunction of the cholinergic system, deposition of amyloid $\beta(A \beta)$ peptide, and hyperphosphorylation of the tau protein $(7,8)$. Due to damage to cognitive function-related neurons, patients with $\mathrm{AD}$ often exhibit progressive memory loss and a decline in other cognitive functions, which manifests as symptoms of progressive cognitive dysfunction $(9,10)$. Functional enrichment analysis of single-nucleotide polymorphisms revealed that FGF14 was elevated in patients with $\mathrm{AD}$ and was subject to phosphorylation by c-Jun N-terminal kinase (JNK). Furthermore, FGF14 messenger RNA (mRNA) was found to be upregulated in patients with early-onset AD (11), suggesting that FGF14 may be implicated in the pathogenesis of $\mathrm{AD}$. However, the mechanism underlying the role of FGF14 in $\mathrm{AD}$ has yet to be reported. Given that the FGF14 protein carries major functional significance within the nervous system, and research on it is still in the initial stage, the establishment of method for generating large amounts of exogenous FGF14 and further exploration of this protein's biological function in vitro and in vivo are of utmost importance.

As mentioned above, the main pathophysiological characteristics of $\mathrm{AD}$ include the deposition of $\mathrm{A} \beta$ peptide and hyperphosphorylation of the tau protein $(7,8)$. A $\beta$ is an insoluble fibril produced via the continuous hydrolysis of amyloid precursor protein (APP) under the action of $\beta$ and $\gamma$-secretases. $A \beta$ precipitates and accumulates in the cell matrix, forming neuritic plaques, and thus leading to neuronal degeneration (12). Once $A \beta$ has accumulated in the synaptic space, it interferes with the associated signaling, resulting in the loss of synaptic function (13). Additionally, $\mathrm{A} \beta$ can induce tau protein phosphorylation by activating the related kinases (14), resulting in microtubule damage and the subsequent formation of neurofibrillary tangles in neurons. Put succinctly, the occurrence of $\mathrm{AD}$ results from complex interplay between a number of factors.

Reducing $A \beta$-induced cytotoxicity may represent an effective approach to treating AD. Owing to synaptic changes, neurotransmitter loss, and neuronal apoptosis within the brain, Patients with $\mathrm{AD}$ often develop cognitive impairment. As a regulator of synaptic excitability, FGF14 can regulate synaptic plasticity and promote neuronal proliferation and differentiation, suggesting its potential as an effective therapeutic agent for neurodegenerative disease. As a potent cytotoxic fragment of $A \beta, A \beta 25-35$ is frequently used to establish experimental models of $\mathrm{AD}$ (15). Therefore, exploration of FGF14's biological activity and mechanism of action in A 325 -35-induced $\mathrm{AD}$ models is of great relevance. To this end, it is necessary to establish a method for obtaining large amounts of highpurity recombinant FGF14.

FGF family members are mainly expressed via Escherichia coli (E. coli) systems. To efficiently obtain a large quantity of rhFGF14 protein with relatively high purity, we optimized the nucleotide sequence of FGF14, constructed the pET15bhFGF14 recombinant plasmid, and transferred it into $E$. coli BL21 (DE3) to express insoluble rhFGF14. Through washing and guanidine hydrochloride denaturation of inclusion bodies, dilution renaturation to restore protein spatial structure, and purification via His-tag expression and nickel column affinity, we finally obtained high-purity FGF14 protein. We observed that rhFGF14 alleviated Aß25-35induced injury in PC12 cells, indicating its neuroprotective effect against $\mathrm{AD}$ and overall potential as a treatment for neurodegenerative disease. We present the following article in accordance with the MDAR reporting checklist (available at https://dx.doi.org/10.21037/atm-21-2492).

\section{Methods}

\section{Reagents and strains}

A $325-35$ was purchased from Sigma-Aldrich (Sigma, USA). PC12 cells were obtained from Shanghai Cell Bank, Chinese Academy of Sciences (Shanghai, China). Expression vector pET-15b was supplied by Zhejiang Key Laboratory of Wenzhou Medical University (Wenzhou, China). The E. coli DH5 $\alpha$ and BL21 (DE3) strains were 
Table 1 Original fibroblast growth factor14 (FGF14) sequence and optimized FGF14 sequence

Original FGF14 sequence
ATGGTGAAACCGGTTCCGCTGTTTCGCCGCACCGATTTCAAACTGCTGCTGTGTAACCATAAAGATCTGTTTTTCTGCGCGTGAGCAAGC
TGCTGGATTGCTTCAGCCCGAAGAGTATGGGTTCCTGTGGAACATTTCAGCAAGGGCACCCACATGCTGCAGTGCCTGTGTGGCAAAA
GCCTGAAGAAAATAAGACCCGACCGACCCGCAGCTGAAAGGCATTGTGACACGCCTGTATTGCCGCCAGGGTTACTACCTGCAAATG
CATCCTGACGGTGCCCTGGATGGCACCAAAGACGACAGCACCAATAGCACCCTGTTCAACCTGATCCCGGTTGGCCTGCGTGTGGTGG
CAATCCAGGGCGTGAAACCGGTCTGTACATCGCCATGAATGGCGAAGGCTACCTGTATCCGAGCGAACTGTTACCCCGGAATGCAAGT
TTAAAGAAAGTGTGTTGAAAATATTATGTGATCTATAGCAGCATGCTGTACCGCCAGCAGGAAAGTGGCCGCGCCTGGTTTCTGGGTCTG
AATAAAGAGGGCCAGGCCATGAAGGGCAATCGCGTGAAGAAGACCAAACCTGCCGCCCATTTCTGCCGAAACCGCTGGAAGTTGCCAT
GTATCGCGAACCGAGCCTGCATGACGTGGGCGAAACCGTTCCGAAACCGGGCGTTACCCCTAGCAAGAGCACCAGCGCAAGTGCCATTA
TGAACGGCGGTAAGCCGGTGAATAAAAGAAACCACCTAA
Optimized FGF14 sequence
ATGGTGAAACCGGTTCCGCTGTTTCGCCGCACCGATTTCAAACTGCTGCTGTGTAACCATAAAGATCTGTTTTTTCTGCGCGTGAGCAAGC
TGCTGGATTGCTTCAGCCCGAAGAGTATGTGGTTCCTGTGGAACATTTCAGCAAGGGCACCCACATGCTGCAGTGCCTGTGTGGCAAAA
GCCTGAAGAAAATAAGAACCCGACCGACCCGCAGCTGAAAGGCATTGTGACACGCCTGTATTGCCGCCAGGGTTACTACCTGCAAATG
CATCCTGACGGTGCCCTGGATGGCACCAAAGACGACAGCACCAATAGCACCCTGTTCAACCTGATCCCGGTTGGCCTGCGTGTGGTGG
CAATCCAGGGCGTGAAACCGGTCTGTACATCGCCATGAATGGCGAAGGCTACCTGTATCCGAGCGAACTGTTACCCCGGAATGCAAGT
TTAAAGAAATGTGTTTAAAATTTTATGTGATCTATAGCAGCATGCTGTACCGCCAGCAGGAAAGTGGCCGCGCCTGGTTTCTGGGTCTG
AATAAAGAGGGCCAGGCCATGAAGGGCAATCGCGTGAAGAAGACCAAACCTGCCGCCCATTTCTGCCGAAACCGCTGGAAGTTGCCAT
GTATCGCGAACCGAGCCTGCATGACGTGGGCGAAACCGTTCCGAAACCGGGCGTTACCCCTAGCAAGAGCACCAGCGCAAGTGCCATTA
TGAACGGCGGTAAGCCGGTGAATAAAAGCAAACCACCTAA

supplied by Solarbio Life Science (Beijing, China).

\section{Construction of the rbFGF14 expression vector}

The pET15b-rhFGF14 recombinant plasmid was constructed by double-digesting the optimized human FGF14 complementary DNA (cDNA) sequence (GenBank accession number: NM_001321931.1) (Table 1) with NdeI and BamHI (TaKaRa, Shanghai) and inserting it into the pET-15b expression vector. Restriction endonuclease assay and DNA sequencing were employed to confirm that cDNA had been successfully inserted into the plasmid.

\section{Inducing the expression of rbFGF14}

The recombinant plasmid was transferred into BL21(DE3) competent cells (Solarbio, Beijing), and the colonies were cultured in lysogeny broth (LB) solid medium containing Amp+ (final concentration: $100 \mu \mathrm{g} / \mathrm{mL}$ ) at a constant temperature of $37^{\circ} \mathrm{C}$. Upon the $\mathrm{A}_{600}$ value reaching $0.8-1.0,1 \mathrm{~mL}$ of the bacterial fluid was collected as a preinduction sample. The inductive agent isopropyl-beta-Dthiogalactoside (IPTG; Dingguo, Beijing) was added to the remaining bacterial liquid to a final concentration of $1 \mathrm{mmol} / \mathrm{L}$. Samples were obtained at 1, 2, 3, and 4 hours after induction and verified via $12 \%$ sodium dodecyl sulphate-polyacrylamide gel electrophoresis (SDS-PAGE) (Yeasen, Shanghai, China). And the rhFGF14 levels were determined by Coomasi Bright Blue Staining. Western blot analysis with rhFGF14 monoclonal antibody (Abcam, United States; diluted at 1:1,000). The colony with the highest expression level of rhFGF14 was used as seed strains for subsequent high-density fermentation.

\section{Propagation}

The seed strains were added to $250 \mathrm{~mL} \mathrm{LB}$ liquid medium containing Amp+ (final concentration: $100 \mu \mathrm{g} / \mathrm{mL}$ ) at a ratio of $1: 20(\mathrm{v} / \mathrm{v})$ before being propagated for 10 hours in a constant-temperature shaker at $37^{\circ} \mathrm{C}$ and $180 \mathrm{rpm}$ until the $\mathrm{A}_{600}$ value reached 3-5. And the seed strains could then be used as second-generation seeds. The secondgeneration seeds were cultured at 1:10 (v/v) under the same conditions. When the $\mathrm{A}_{600}$ value reached $0.8-1.0,1 \mathrm{~mL}$ the bacterial fluid was collected as a pre-induction sample, and IPTG was added to the remaining bacterial solution to a final concentration of $1 \mathrm{mmol} / \mathrm{L}$. Protein expression was detected via $12 \%$ SDS-PAGE and determined by Coomasi Bright Blue Staining for the respective time points under 2 culture conditions: shaking culture at $32{ }^{\circ} \mathrm{C}$ and $180 \mathrm{rpm}$ for 4 hours, and shaking culture at $18^{\circ} \mathrm{C}$ and $180 \mathrm{rpm}$ for 24 hours. After induction, the solution was centrifuged at 
$4{ }^{\circ} \mathrm{C}$ and $9,000 \mathrm{rpm}$ for 15 minutes, and the bacteria were collected and frozen at $-80^{\circ} \mathrm{C}$.

\section{Solubility identification}

The bacteria with induced expression of rhFGF14 at 2 different temperatures were thawed, resuspended by adding lysis buffer at a ratio of 1:40 (v/v), and ultrasonicated twice at frame rates of $40 \%, 50 \%$, and $60 \%$, respectively. After ultrasonication, the solution was centrifuged at $4{ }^{\circ} \mathrm{C}$ and $12,000 \mathrm{rpm}$ for 30 minutes. The supernatant was then collected and precipitated, and protein solubility was determined via $12 \%$ SDS-PAGE.

\section{Inclusion body washing}

The target protein was observed to mainly be expressed in the form of inclusion bodies. The recombinant protein was centrifuged, and the precipitate was added into wash buffer I (20 mmol/L TRIS hydrochloride (Tris-HCL), $0.1 \mathrm{~mol} / \mathrm{L}$ Sodium chloride $(\mathrm{NaCl}), 1 \%(\mathrm{v} / \mathrm{v})$ Triton-X-100, $0.5 \%$ sodium deoxycholate) at a ratio of $1: 40(\mathrm{v} / \mathrm{v})$. After washing, the precipitate was collected by centrifuging the solution at $4{ }^{\circ} \mathrm{C}$ and $12,000 \mathrm{rpm}$ for 30 minutes. The protein was then washed with wash buffer II $[20 \mathrm{mmol} / \mathrm{LTris}-\mathrm{HCL}$, $0.1 \mathrm{~mol} / \mathrm{LNaCl}, 1 \%$ (v/v) Triton-X-100, $2 \mathrm{~mol} / \mathrm{L}$ urea], and the precipitate was collected via centrifugation under the same conditions.

\section{Renaturation of inclusion body protein}

The precipitate was added to denaturation buffer [20 mmol/L Tris-HCL, $0.05 \mathrm{~mol} / \mathrm{L} \mathrm{NaCl,}$ $1 \%(\mathrm{v} / \mathrm{v})$ Triton-X-100, $5 \%$ glycerol, $7 \mathrm{~mol} / \mathrm{L}$ guanidine hydrochloride] at a ratio of 1:40 (v/v), left to denature overnight, and then centrifuged at 20,000 rpm for 30 minutes, after which the supernatant was collected. After stirring the denatured solution for 16-20 hours, renaturation diluent $[20 \mathrm{mmol} / \mathrm{L}$ Tris-HCL, $0.15 \mathrm{~mol} / \mathrm{L} \mathrm{NaCl}, 1 \%$ (v/v) Triton-X-100, $5 \%$ glycerol] was added to dilute it, and the supernatant was collected via filtration through a $0.45-\mu \mathrm{m}$ membrane. All of the above operations were performed at $4{ }^{\circ} \mathrm{C}$.

\section{Protein purification}

Loading buffer ( $20 \mathrm{mmol} / \mathrm{L}$ Tris-HCL, $0.15 \mathrm{~mol} / \mathrm{L} \mathrm{NaCl}$ ) was used to balance the nickel column, and renaturation solution was pumped into the nickel column for affinity. Under an ultraviolet wavelength of $280 \mathrm{~nm}$, the nickel column was eluted with imidazole at concentrations of 50 , $100,150,250$, and $500 \mathrm{mmol} / \mathrm{L}$. The eluted samples were collected and analyzed via $12 \%$ SDS-PAGE. Purification of the ultrafiltration-concentrated protein was achieved using a 15 (KD) ultrafiltration tube; the imidazole was removed; and the protein concentration was determined using a bicinchoninic acid (BCA) assay kit (Beyotime, Shanghai, China). All of the above operations were performed at $4{ }^{\circ} \mathrm{C}$.

\section{Aß25-35 toxicity in PC12 cells}

PC12 cells were put in 96-well plates with $8 \times 10^{3}$ cells/well. They were cultured in Dulbecco's Modified Eagle Medium (DMEM; Gibco, China) containing 10\% fetal bovine serum (FBS; Gibco, USA) for 24 hours, and then starved for 24 hours. Thereafter, cells cultured with different concentrations of A $\beta 25-35$ (5, 10, 20, or $40 \mu \mathrm{mol} / \mathrm{L})$ for 24 hours or 48 hours were assessed as the experimental groups. An equal volume of phosphate-buffered saline (PBS) was added as the blank group, and PC12 cells cultured under the same conditions as the experimental group served as the control group. Cellular proliferative activity was determined via Cell Counting Kit-8 (CCK-8) assays, in which cell survival rate $=(\mathrm{A}$ experimental group - A blank group)/(A control group - A blank group). The concentration and time required for A $225-35$-induced injury were determined on the basis of the cell survival rate for treated PC12 cells.

\section{Proliferation-promoting effect of rhFGF14 on AB25-35- treated PC12 cells}

PC12 cells were put in 96-well plates with $8 \times 10^{3}$ cells/ well. They were cultured for 24 hours, A $25-35$ at the optimal dose, as determined via toxicity assessment, was coincubated with FGF14. Thereafter, rhFGF14 was added at different concentrations, and the cell survival rate was detected using a CCK-8 kit (Dojindo, Kumamoto, Japan). The cell culture conditions were the same as those used for the toxicity test.

\section{Detection of lactate dehydrogenase and malondialdebyde levels in the culture medium}

The levels of lactate dehydrogenase (LDH) and malondialdehyde (MDA) in the cell culture medium were 
detected using LDH and MDA kits (Jiancheng, Nanjing), respectively. The supernatant was obtained through centrifugation of the cell culture solution, and the LDH and MDA levels were determined as per the manufacturer's instructions.

LDH activity $(\mathrm{M} / \mathrm{L})=($ measured $\mathrm{OD}$ value - control OD value/standard OD value - blank OD value) $\times$ concentration of standard substance $(2 \mathrm{mmol} / \mathrm{L}) \times 1,000$

MDA content $(\mathrm{nmol} / \mathrm{mL})=($ measured OD value control OD value/standard OD value - blank OD value) $\times$ concentration of standard substance $(10 \mathrm{nmol} / \mathrm{L}) \times$ dilution multiple of the sample before test

\section{Calcein-acetoxymethyl/propidium iodide fluorescence double staining}

The methyl acetate of calcein-acetoxymethyl (AM) is highly lipophilic, which allows for cell membrane penetration. Once within cells, the calcein-acetoxymethyl (AM) group is removed by esterase, and the generated calcein emits strong green fluorescence. The propidium iodide (PI) nuclear dye cannot pass through the membrane of living cells, but it can enter dead cells via the damaged cell membrane and intercalate DNA, thus producing red fluorescence. Briefly, cells were centrifuged and then resuspended in PBS, and this step was repeated several times for esterase removal. The cell suspension and calcein-AM staining solution (Dojindo, Kumamoto, Japan) were mixed and cultured at $37{ }^{\circ} \mathrm{C}$. Live cells (green fluorescence) and dead cells (red fluorescence) were observed under a fluorescence microscope.

\section{Annexin V-FITC/PI}

In normal cells, phosphatidylserline (PS) is distributed on the inner side of the cell membrane lipid bilayer, but in apoptotic cells, it accumulates on the membrane's outer side. As Annexin V has high affinity for PS, it binds to the cell membrane of early-stage apoptotic cells via the exposed PS. Briefly, cells were added into buffer solution and mixed well, and then the addition of Annexin V-FITC was made. Finally, PI staining solution was added, and apoptotic cells were detected via flow cytometry.

\section{Western blot}

Treated PC12 cells treated were collected from 6-well plates, lysed in radioimmunoprecipitation assay (RIPA) buffer, and centrifuged at $4{ }^{\circ} \mathrm{C}$ and $12,000 \mathrm{rpm}$ to collect the supernatant. BCA protein analysis kit was used to determine the protein concentration. After being separated via $12 \%$ SDS-PAGE, the protein was transferred to a polyvinylidene fluoride (PVDF) membrane and blocked with $5 \%$ skim milk for 60 minutes. The membrane was incubated at $4{ }^{\circ} \mathrm{C}$ with the following primary antibodies: anti-JNK, anti-Phosphoc-Jun N-terminal kinase(P-JNK), anti-extracellular signalregulated kinase 1/2 (ERK1/2), anti-Phospho-extracellular signal-regulated kinase 1/2 (P-ERK1/2), anti-p38, antiPhospho-p38 (P-p38), and anti-Tubulin (all Cell Signaling Technology, Beverly, MA, United States; diluted at 1:1,000). After that, the membrane was incubated with secondary antibodies (goat anti-rabbit antibodies; 1:1,000). After 3 washes with tris-buffered saline with Tween, the protein bands were visualized using an electrochemiluminescence kit (Thermo scientific, NY, USA).

\section{Statistical analysis}

The data were expressed as mean \pm standard error of the mean (SEM). Student's $t$-test was adopted for comparisons between 2 groups. A comparison of multi-group data was carried out with a One-way analysis of variance (ANOVA) followed by Dunnett's test. Statistical significance was considered at either the $\mathrm{P}<0.05$ or $\mathrm{P}<0.01$ level.

\section{Results}

\section{Vector construction and expression of rbFGF14}

The fragment size of the FGF14 gene double-digested by NdeI and BamHI was approximately $750 \mathrm{bp}$, while that of the pET-15b vector was about 5,700 bp (Figure 1A); these sizes were in line with those expected, indicating that the FGF14 gene had been successfully inserted into the pET-15b expression vector. The result was confirmed via DNA sequencing. The positive recombinant plasmid was transferred into BL21 (DE3) cells, and expression of rhFGF14 was induced with $1 \mathrm{mmol} / \mathrm{L}$ IPTG at $37^{\circ} \mathrm{C}$ for 4 hours. Samples were collected and analyzed via $12 \%$ SDSPAGE. The different single colonies exhibited significant protein expression bands after induction compared with before induction (Figure 1B). Western blot analysis confirmed that the induced protein was indeed rhFGF14 (Figure 1C).

The single colony with highest expression, as shown in Figure 1B, was selected as the seed strain, and the 
A

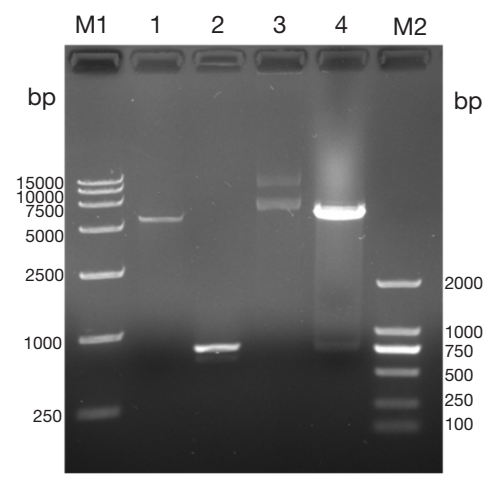

B

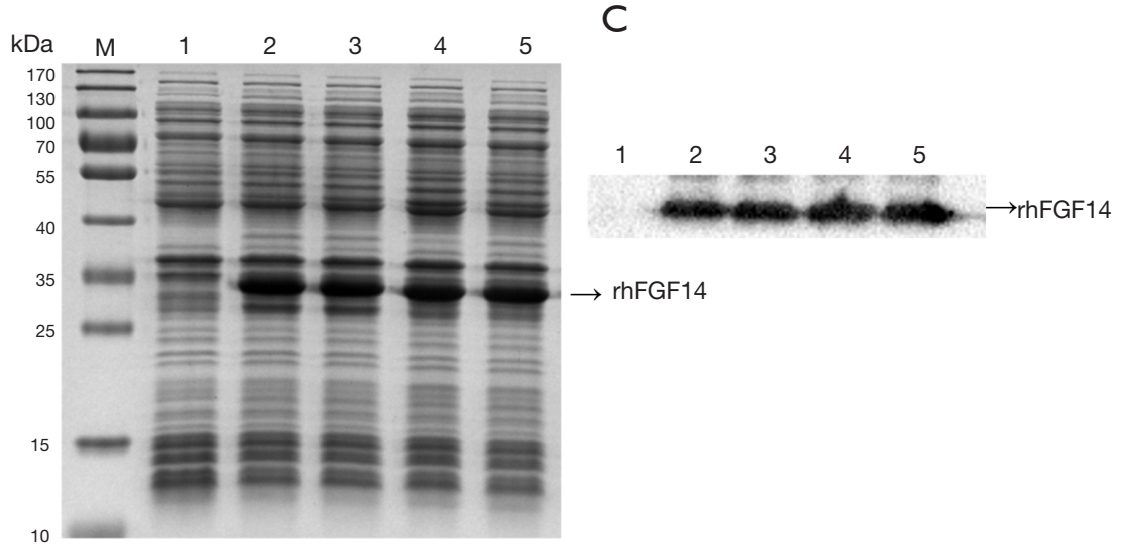

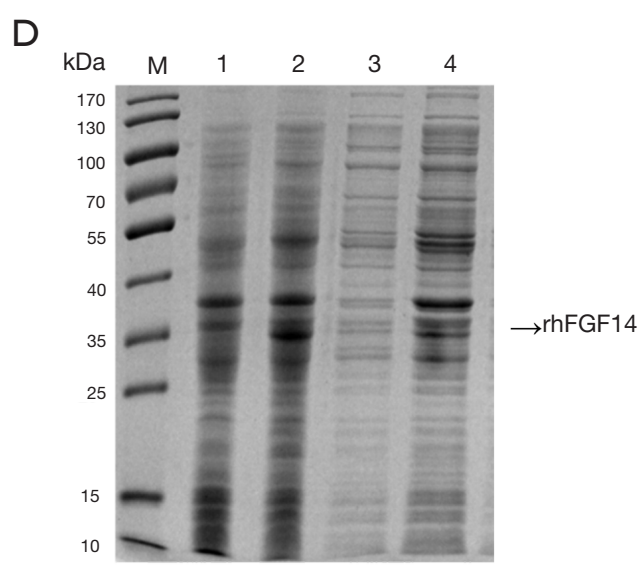

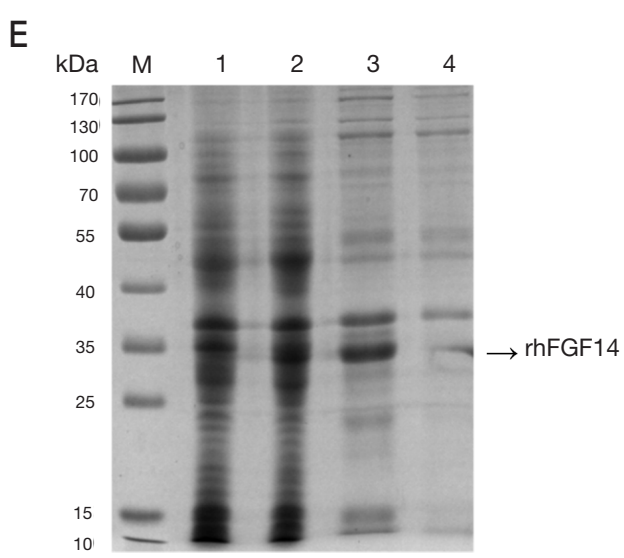

Figure 1 Vector construction and recombinant human fibroblast growth factor 14 (rhFGF14) expression. (A) Electrophoresis detection of the enzyme-digested pET15b-rhFGF14 recombinant vector. 1: pET-15b vector; 2: FGF14 cDNA; 3: pET15b-rhFGF14 recombinant vector; 4: double-digested product of pET15b-rhFGF14 (the enzyme digestion sites were BamHI and NdeI); M1: DNA Marker DL 15,000; M2: DNA Marker DL 2,000. (B) 12\% (v/v) sulphate-polyacrylamide gel electrophoresis (SDS-PAGE) analysis of induced rhFGF14 expression. 1: Before induction; 2,3,4,5: different single colonies after induction via isopropyl-beta-D-thiogalactoside (IPTG); M: protein molecular weight standard. (C) Western blot detection of induced rhFGF14 expression. 1: Before induction; 2,3,4: different single colonies after induction via IPTG. (D) $12 \%$ (v/v) SDS-PAGE analysis of rhFGF14 solubility with $4 \mathrm{~h}$ induction at $32{ }^{\circ} \mathrm{C}, 180 \mathrm{rpm}$, IPTG final concentration of $1 \mathrm{mmol} / \mathrm{L}$. 1: Total protein in BL21 (DE3) cells before induction; 2: total protein in BL21 (DE3) cells after induction; 3: supernatant after expression induction and lysis; 4: precipitate after expression induction and lysis; M: protein molecular weight standard. (E) $12 \%(\mathrm{v} / \mathrm{v})$ SDS-PAGE analysis of rhFGF14 solubility with $24 \mathrm{~h}$ induction at $18^{\circ} \mathrm{C}, 180 \mathrm{rpm}$, IPTG final concentration of $1 \mathrm{mmol} / \mathrm{L}$. 1: Total protein in BL21 (DE3) cells before induction; 2: total protein in BL21 (DE3) cells after induction; 3: precipitate after expression induction and lysis; 4: supernatant after expression induction and lysis; M: protein molecular weight standard.

fermentation medium and culture temperature were optimized for propagation. Two different culture conditions were established. Figure $1 D$ shows SDS-PAGE analysis after induction for 4 hours at $32{ }^{\circ} \mathrm{C}$ under $180 \mathrm{rpm}$, and a final IPTG concentration of $1 \mathrm{mmol} / \mathrm{L}$. Figure $1 \mathrm{E}$ shows SDSPAGE analysis after induction for 24 hours at $18{ }^{\circ} \mathrm{C}$ under $180 \mathrm{rpm}$, and a final IPTG concentration of $1 \mathrm{mmol} / \mathrm{L}$. The solubility determination results indicated that the target protein still existed in the form of inclusion bodies after the induction conditions had been changed, and soluble protein could not be produced. Cells were therefore cultured at $32{ }^{\circ} \mathrm{C}$ under $180 \mathrm{rpm}$ until the $\mathrm{A}_{600}$ value reached $0.8-1.0$, and the expression of rhFGF14 was induced for 4 hours after the addition IPTG (final concentration: $1 \mathrm{mmol} / \mathrm{L}$ ), as the optimal fermentation conditions for rhFGF14 protein expression. 
A

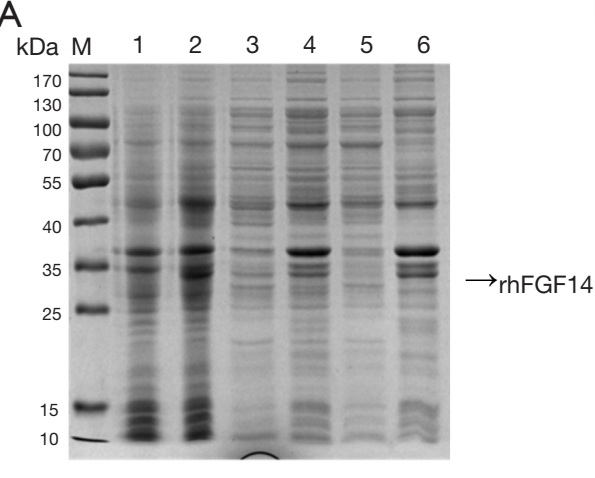

B

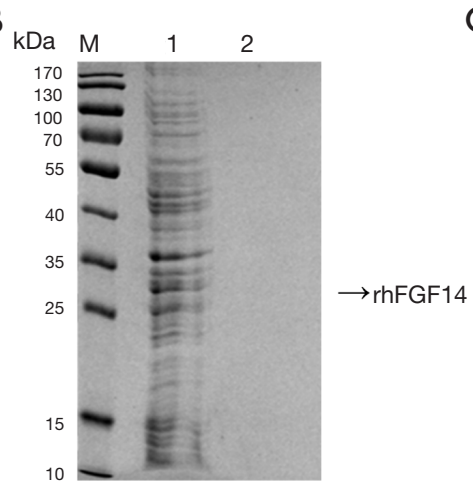

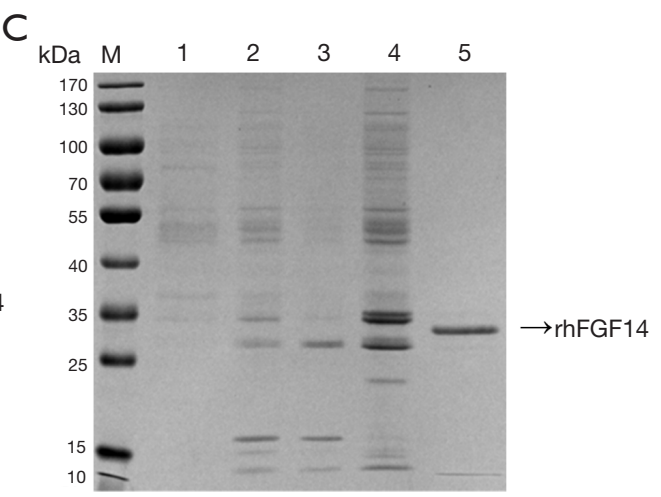

Figure 2 Renaturation and purification of rhFGF14 inclusion bodies. (A) 12\% (v/v) SDS-PAGE analysis of rhFGF14 inclusion bodies after washing. 1: Total protein in bacteria before induction; 2: total protein in bacteria after induction; 3 : the supernatant from the first round of washing after lysing of the bacteria; 4: precipitate from the first round of washing after lysing of the bacteria; 5: supernatant from the second round of washing after lysing of the bacteria; 6 . precipitate from the second round of washing after lysing of the bacteria; M: protein molecular weight standard. (B) 12\% (v/v) SDS-PAGE analysis of rhFGF14 inclusion bodies after denaturation. 1: Denaturation supernatant after expression induction and lysis; 2: Denaturation precipitate after expression induction and lysis; M: protein molecular weight standard. (C) 12\% (v/v) SDS-PAGE analysis of Ni column purification of rhFGF14. 1: Dilution and renaturation after expression induction and lysis; 2: elution with $50 \mathrm{mM}$ imidazole; 3: elution with $100 \mathrm{mM}$ imidazole; 4: elution with $250 \mathrm{mM}$ imidazole; 5: elution with $500 \mathrm{mM}$ imidazole; $\mathrm{M}$ : protein molecular weight standard.

\section{Renaturation and purification of rbFGF14 inclusion bodies}

We found that rhFGF14 protein mainly existed in the form of inclusion bodies, and observed considerable protein impurity in the precipitate of lysed bacteria. We washed the inclusion body protein repeatedly with wash buffer, and examined the supernatant and precipitate samples via SDS-PAGE after each round of washing. The protein in wash precipitate was purer than before washing. (Figure $2 A$ ). While some of the impure protein had been washed away, rhFGF14 protein still existed in the form of inclusion bodies. Thus, the inclusion body protein was denatured with $7 \mathrm{M}$ guanidine hydrochloride at $4{ }^{\circ} \mathrm{C}$ overnight. After centrifugation, the supernatant was obtained, and we observed that the inclusion bodies had been completely dissolved (Figure 2B). By optimizing the dilution conditions, the supernatant was diluted 30 times and stirred continuously for 18 hours to complete the dilution and renaturation processes. As the His-tag of vector pET15b has high affinity for the nickel column, we bound the renatured solution to the nickel column and eluted it via gradient elution with $50,100,250$, and $500 \mathrm{mmol} / \mathrm{L}$ imidazole. As shown in Figure $2 C$, at $500 \mathrm{mM}$, rhFGF14 protein was completely eluted. The eluted protein was desalted and concentrated via ultrafiltration, which yielded
rhFGF14 protein of high purity.

\section{The neuroprotective effect of rbFGF14 on AB25-35- injured PC12 cells}

PC12 cells have the morphology and function of neuronlike cells; therefore, we established our AD model by treating PC12 cells with A $\beta 25-35$ (5, 10, 20, and $40 \mu \mathrm{mol} / \mathrm{L}$ for 24 or 48 hours). The survival rate of cells decreased gradually in a time- and dose-dependent manner (Figure 3A). Subsequently, $40 \mu \mathrm{mol} / \mathrm{L}$ A $\beta 25-35$ and 48 hours were selected as the treatment conditions for establishing the $\mathrm{AD}$ model in the following experiments.

We added rhFGF14 $(1,2,4,8,16,32,64,128$, and $256 \mathrm{ng} / \mathrm{mL}$ ) to the established disease model to assess this recombinant protein's neuroprotective effects. The results indicated that rhFGF14 could alleviate A 25 -35-induced PC12 cell injury, as indicated by an increased survival rate (Figure 3B).

$\mathrm{LDH}$, which is abundant in the cytoplasm, is released from cells following damage or cell death. MDA is a product of lipid peroxide degradation, and its levels can reflect the degree of lipid peroxidation, thus providing an indirect measure of cell injury. The LDH and MDA detection results revealed that rhFGF14 could significantly, 
A

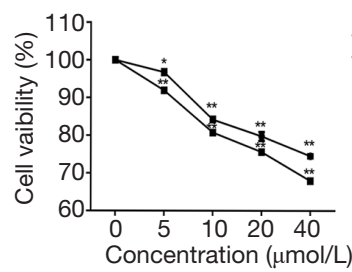

C

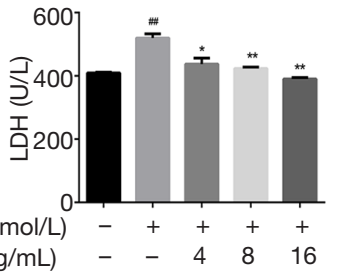

$\mathrm{D}$ $\mathrm{A} \beta_{25-35}(40 \mu \mathrm{mol} / \mathrm{L})$
rhFGF14 $(\mathrm{ng} / \mathrm{mL})$

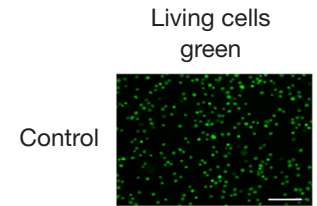

$\mathrm{A} \beta_{25-35}$ only

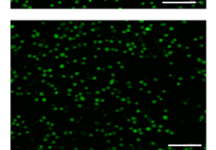

$\mathrm{A} \beta_{25-35}+4 \mathrm{ng} / \mathrm{mL}$ FGF14

$\mathrm{AB}_{25-35}+8 \mathrm{ng} / \mathrm{mL}$ FGF14

$\mathrm{A} \beta_{25-35}+16 \mathrm{ng} / \mathrm{mL}$ FGF14
$-48 \mathrm{~h}$

Dead cells
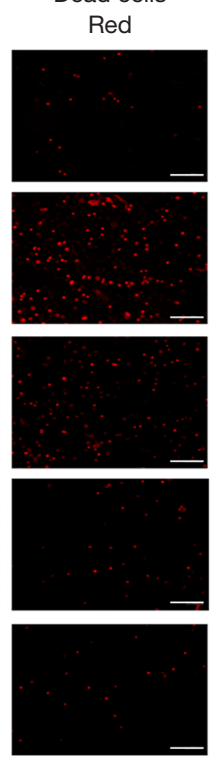

B

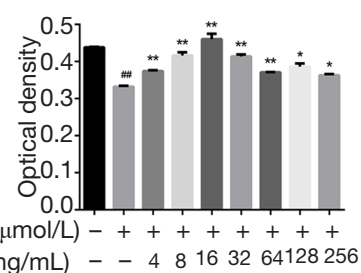

rhFGF14 (ng/mL) - - 48163264128256

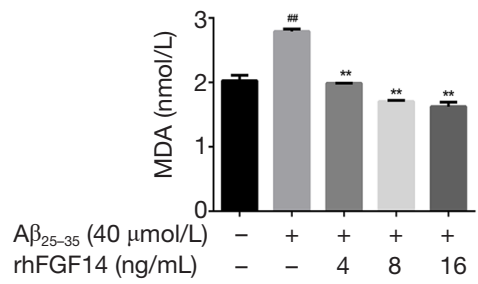

$\mathrm{A} \beta_{25-35}(40 \mu \mathrm{mol} / \mathrm{L})$

$$
\text { Merge }
$$
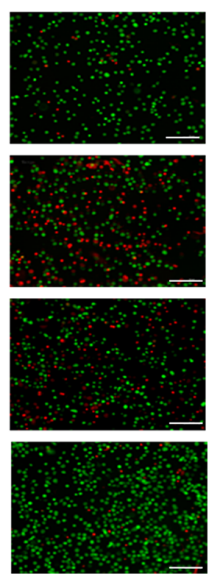

$A \beta_{25-35}(40 \mu \mathrm{mol} / \mathrm{L})$ rhFGF14 (ng/mL) - $\quad-\quad \begin{array}{llll}+ & 4 & 8 & 16\end{array}$
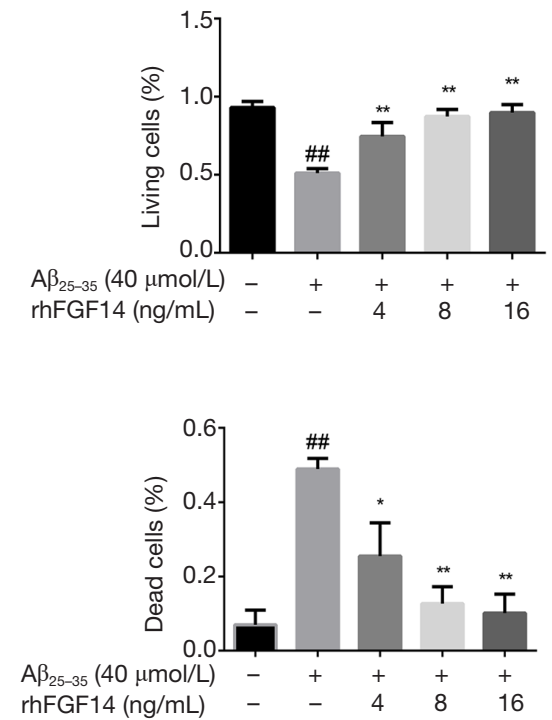

rhFGF14 (ng/mL)

\section{$E$}

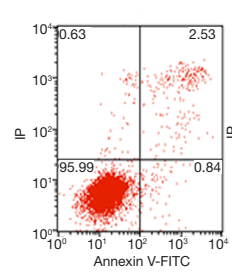

Control

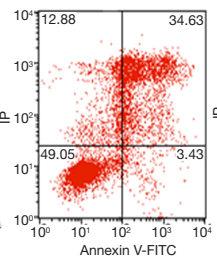

Model

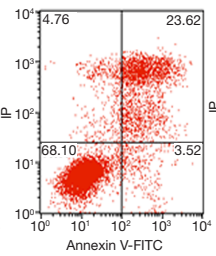

FGF14 $(4 \mathrm{ng} / \mathrm{mL})$

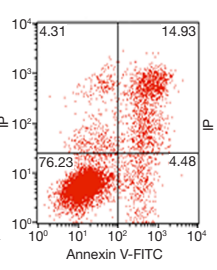

FGF
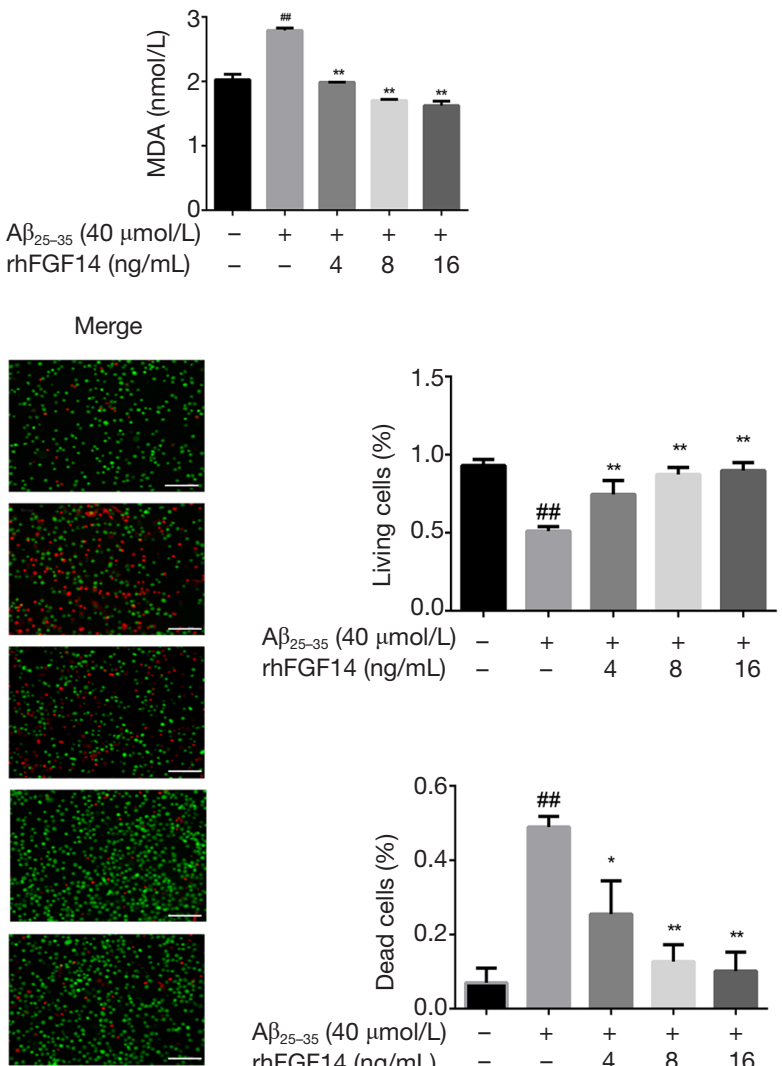

Figure 3 The neuroprotective effect of rhFGF14 in A 25-35-injured PC12 cells. (A) Cell Counting Kit-8 (CCK8) assay was used to determine the survival rate of PC12 cells treated with different concentrations of Aß25-35 for different lengths of time. (B) CCK8 assay was used to assess the effect of rhFGF14 at different concentrations on A325-35-injured PC12 cells; (C) Lactate dehydrogenase (LDH) release and malondialdehyde (MDA) content were determined in the A 25 -35 model group and rhFGF14-treated cells; (D) the neuroprotective effect of rhFGF14 in Aß25-35-treated PC12 cells was detected via calcein-acetoxymethyl/propidium iodide (calcein-AM/PI) double staining. Scale bar $=100 \mu \mathrm{m}$. (E) Annexin V-FITC/PI staining was used for flow cytometry detection of apoptosis to examine the effect of rhFGF14 on A 25-35-treated PC12 cells. Data were taken as the mean \pm SD of 3 independent experiments. '**** indicates a significant difference relative to the control at $\mathrm{P}<0.05, \mathrm{P}<0.01$; and ${ }^{\left({ }^{*} \prime \prime\right.}$ indicates a significant difference relative to the model at $\mathrm{P}<0.01$. 

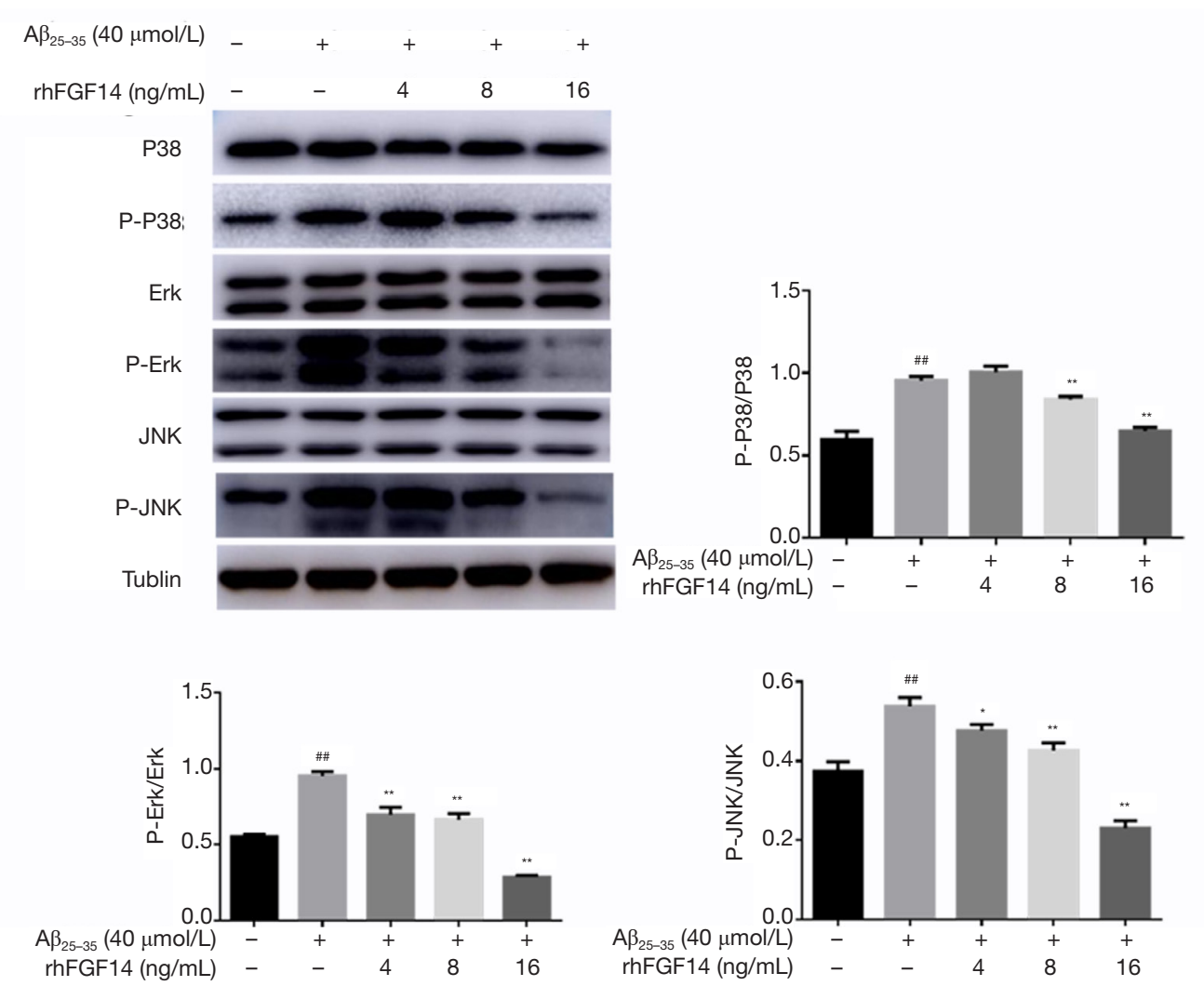

Figure 4 Neuroprotective effect of rhFGF14 in Aß25-35-injured PC12 cells. The levels of total and phosphorylated Phospho-p38 (P38), extracellular signal-regulated kinase1/2 (ERK), and c-Jun N-terminal kinase (JNK) in the Alzheimer's disease (AD) model were detected by western blot. Data are presented as the mean \pm SD of 3 independent experiments. '***' indicates a significant difference from the model at $\mathrm{P}<0.05, \mathrm{P}<0.01$; ${ }^{\text {(⿻\#, }}$ indicates a significant difference from the control at $\mathrm{P}<0.01$.

dose-dependently reduce the degree of A $\beta 25$-35-induced PC12 injury (Figure 3C). Fluorescence microscopy with calcein-AM/PI indicated that a large number of cells died following A $325-35$ treatment, while the number of dead cells dropped significantly under simultaneous rhFGF14 treatment (Figure 3D). Annexin V-FITC/PI flow cytometry analysis demonstrated that rhFGF14 dose-dependently reduced apoptosis of PC12 cells (Figure $3 E$ ). To sum up, rhFGF14 protected PC12 cells against A 325 -35-induced injury.

\section{rhFGF14 inhibited the mitogen-activated protein kinase (MAPK) signaling patbway}

To further explore the mechanism of neuroprotective activity of rhFGF14 in the AD model, its effect on MAPK signaling activation was assessed. Western blot indicated that the MAPK pathway was activated following A 325 -
35 treatment, with the phosphorylation levels of P38, ERK, and JNK increasing significantly. Treatment with FGF14 (4, 8, and $16 \mathrm{ng} / \mathrm{mL})$ suppressed A $\beta 25$ 35 -induced phosphorylation of all 3 kinases in a dosedependent manner (Figure 4). These results indicated that $\mathrm{A} \beta$ accumulation activates MAPK signaling, which can be inhibited by rhFGF14, suggesting that rhFGF14 may exert its neuroprotective effect via the inhibition of MAPK signaling.

\section{Discussion}

In the current work, we optimized the FGF14 sequence, constructed the pET15b-rhFGF14 recombinant plasmid, and determined the optimal conditions for high-yield expression of rhFGF14 in an E. coli system. The Histagged rhFGF14 protein was obtained through renaturing and purification of inclusion bodies via nickel affinity 
column chromatography. The addition of rhFGF14 significantly alleviated A $\beta$-induced PC12 cell injury. Further mechanistic experiments revealed that rhFGF14 exerted its neuroprotective effect via the inhibition of MAPK activation. MAPK suppression may in turn alleviate cellular injury by downregulating inflammatory and apoptotic responses triggered by the accumulation of $A \beta$.

FGF14 has close associations with cognitive and mental disorders, such as neurodegenerative disease, schizophrenia, anxiety disorder, and depression (11). FGF14 is highly expressed in the brain, and in Purkinje cells, it interacts with voltage-gated $\mathrm{Nav}$ channels to regulate neuronal excitability. FGF14 also plays an important role in synaptic plasticity and hippocampal neurogenesis via its regulation of synaptic transmission from granule cells to Purkinje cells (16). FGF14 gene deletion has been reported to suppress neuronal proliferation, development, and maturation, as well as neurogenesis (17). These observations suggest that FGF14 plays a central role in complex brain disease etiology, and thus, may have potential as a therapeutic agent for neurological conditions. Therefore, large-scale production of FGF14 protein and a deeper understanding of its effect in neurological disease are of major importance. To this end, we sought to establish a system for recombinant FGF14 production and assess the protein's effect in a model of $\mathrm{AD}$.

High rhFGF14 protein expression was achieved through optimization of the fermentation process in E. coli. However, rhFGF14 was mainly produced in the form of inclusion bodies. The formation of protein inclusion bodies results from an imbalance between the proper folding, aggregation, and degradation of protein (18). The main reason for the formation of rhFGF14 inclusion bodies might be that the protein expression rate was excessively high, leaving the host cells no time to modify and adequately fold the protein. Consequently, misfolded protein is produced and polymerizes to form inclusion bodies. In this study, we sought to reduce protein expression by optimizing culture conditions; however, the results were unsatisfactory, as inclusion bodies remained (Figure 1D,E). Therefore, we further purified rhFGF14 protein through renaturation of inclusion bodies.

Renaturation is a key step in protein purification. Currently, commonly employed renaturation methods include dilution renaturation, dialysis renaturation, and column renaturation. In our experiment, $8 \mathrm{M}$ urea was initially used to dissolve the inclusion bodies, but its efficiency was unexpectedly low. In contrast, $7 \mathrm{M}$ guanidine hydrochloride was able to dissolve the inclusion bodies completely, implying that column renaturation would be difficult to implement. Reducing the interaction among molecules and lowering the protein concentration are prerequisites for inhibiting protein aggregation and increasing refolding during renaturation (19). Dialysis renaturation demands a large amount of buffer and has the disadvantage of low renaturation yield owing to protein aggregation; thus, it is not suitable for the renaturation of large quantities of inclusion bodies. Finally, we adopted the method of dilution renaturation. In addition to optimizing the renaturation efficiency by adjusting the dilution rate and ratio, we added glycerol to prevent protein collision and aggregation. FGF has a high affinity for heparin and can specifically bind to the heparan sulfate (HS) receptor. Therefore, most FGF purification methods entail column chromatography with heparin. However, the high affinity for heparin may result in the presence of other impurities in the final product. Fusion tags provide affinity sites, which greatly simplifies the purification process, in turn improving protein yield (20). His-tags do not form dimers during purification and thus do not affect protein properties. Our pET15b vector expressed its own Histag, allowing for specific binding of recombinant protein to the nickel column followed by washing with gradient imidazole to obtain high-purity rhFGF14 (Figure 2C) (21). Overall, fusion tags may hold promise for the purification of recombinant FGF.

As previously stated, the deposition of $\mathrm{A} \beta$ peptide and the hyperphosphorylation of the tau protein are considered to be the major underlying causes of $\operatorname{AD}(7,8)$. In addition to neuritic plaque (NP) and neurofibrillary tangles, the insoluble aggregation of $A \beta$ can trigger peripheral microglia infiltration, which in turn leads to the continuous secretion of inflammatory factors and further aggravates degenerative neuronal injury (22). One previous study reported extensive oxidative stress in patients with $\mathrm{AD}$ (23). Oxidative stress refers to the production of reactive oxygen species (ROS) to an extent that overwhelms cellular antioxidant defense systems, resulting in a redox imbalance, cellular functional damage, or apoptosis. $A \beta$ and tau cause neuronal oxidative injury by compromising the cellular energy supply and antioxidant defense (24). We successfully established an $\mathrm{AD}$ model by inducing PC12 cell injury via treatment with A $325-35$, a key pathogenic factor of AD. Treatment with rhFGF14 indicated that it could exert a neuroprotective effect by significantly reducing the degree of A $\beta 25-35$ induced PC12 cell injury (Figure 3). 
Previous research has established MAPK as a key enzyme in $A \beta$-mediated neurotoxicity, with the MAPK signaling pathway being dysregulated during the pathogenesis of $\mathrm{AD}$ (25). The cascade includes ERK, p38, and JNK. In response to stimulation by various extracellular factors, MAPK signaling drives the pathogenesis of various neurodegenerative diseases via its regulation of cellular processes, including proliferation, differentiation, growth, and apoptosis. Further, the chronic deposition of $A \beta$ leads to mitochondrial dysfunction, consequent oxidative stress, p38 activation, and tau protein hyperphosphorylation (25). In activated microglia, p38 signaling facilitates the production of tumor necrosis factor-alpha (TNF- $\alpha$ ), cyclooxygenase-2 (COX2 ), and inducible nitric oxide synthase to mediate neuroinflammation (26), representing a potential underlying mechanism of $\mathrm{A} \beta$ in $\mathrm{AD}$. The level of phosphorylated JNK has been reported to be upregulated in the postmortem brain samples of patients with $\mathrm{AD}$, co-localizing with $\mathrm{A} \beta(27,28)$. $\mathrm{A} \beta$ can inhibit the expression of antiapoptotic proteins such as $\mathrm{B}$-cell lymphoma-extra large (Bcl-xL) and B-cell lymphoma-2 (Bcl-2) by activating JNK, thus promoting apoptosis to mediate its neurotoxic effects. P-JNK phosphorylates amyloid precursor protein at Thr668; through this, it further promotes $\mathrm{A} \beta$ hydrolysis and the production of $\mathrm{A} \beta$ toxic fragments in the brain parenchyma (29). ERK activation contributes to synaptic plasticity and memory formation (30,31). Nevertheless, aberrant ERK activation can accelerate the formation of neurofibrillary tangles, leading to hippocampal function impairment and memory deficits in patients with $\mathrm{AD}$. Our data confirmed that rhFGF14 could suppress A 325 35 -induced P38, ERK, and JNK phosphorylation (Figure 4), indicating that the neuroprotective effect of FGF14 may be mediated via suppression of the MAPK pathway. Whether neuroprotective effect of FGF14 was suppressed by the MAPK agonist need further investigate. Notably, our present findings are limited to the cellular level. It is therefore essential to explore whether FGF14 exerts neuroprotective effects in animal models of Alzheimer's disease. Further in-depth investigation of its specific underlying mechanism is also required.

\section{Conclusions}

Our study established a simple and efficient process for the production of high-purity rhFGF14, which may represent an effective strategy for recombinant FGF protein production and purification in general. We further demonstrated the neuroprotective effect of FGF14 in an $\mathrm{AD}$ model which was mediated via the inhibition of MAPK signaling. Our current findings provide a basis for further exploration of the biological function of FGF14 in neurodegenerative disease, which may in turn pave the way for the development of FGF14-based therapeutics. Nevertheless, further research is still required to elucidate the exact biological function and underlying mechanism of FGF14.

\section{Acknowledgments}

We thank Dr. Alan K. Chang (Wenzhou University) for his valuable work in revising the language of the manuscript. Funding: The work was supported by the Scientific and Technological Plan Project of Wenzhou (N20180002).

\section{Footnote}

Reporting Checklist: The authors have completed the MDAR reporting checklist. Available at https://dx.doi. org/10.21037/atm-21-2492

Data Sharing Statement: Available at https://dx.doi. org/10.21037/atm-21-2492

Conflicts of Interest: All authors have completed the ICMJE uniform disclosure form (available at https://dx.doi. org/10.21037/atm-21-2492). The authors have no conflicts of interest to declare.

Ethical Statement: The authors are accountable for all aspects of the work in ensuring that questions related to the accuracy or integrity of any part of the work are appropriately investigated and resolved.

Open Access Statement: This is an Open Access article distributed in accordance with the Creative Commons Attribution-NonCommercial-NoDerivs 4.0 International License (CC BY-NC-ND 4.0), which permits the noncommercial replication and distribution of the article with the strict proviso that no changes or edits are made and the original work is properly cited (including links to both the formal publication through the relevant DOI and the license). See: https://creativecommons.org/licenses/by-nc-nd/4.0/.

\section{References}

1. Beenken A, Mohammadi M. The FGF family: biology, 
pathophysiology and therapy. Nat Rev Drug Discov 2009;8:235-53.

2. Laezza F, Gerber BR, Lou JY, et al. The FGF14(F145S) mutation disrupts the interaction of FGF14 with voltagegated $\mathrm{Na}+$ channels and impairs neuronal excitability. J Neurosci 2007;27:12033-44.

3. Teive HAG, Arruda WO. Cognitive dysfunction in spinocerebellar ataxias. Dement Neuropsychol 2009;3:180-7.

4. Gadelha A, Ota VK, Cano JP, et al. Linkage replication for chromosomal region 13q32 in schizophrenia: evidence from a Brazilian pilot study on early onset schizophrenia families. PLoS One 2012;7:e52262.

5. Hagihara H, Takao K, Walton NM, et al. Immature dentate gyrus: an endophenotype of neuropsychiatric disorders. Neural Plast 2013;2013:318596.

6. Bondi MW, Edmonds EC, Salmon DP. Alzheimer's Disease: Past, Present, and Future. J Int Neuropsychol Soc 2017;23:818-31.

7. Gandy S, DeKosky ST. Toward the treatment and prevention of Alzheimer's disease: rational strategies and recent progress. Annu Rev Med 2013;64:367-83.

8. Risacher SL, Saykin AJ. Neuroimaging and other biomarkers for Alzheimer's disease: the changing landscape of early detection. Annu Rev Clin Psychol 2013;9:621-48.

9. Stokin GB, Lillo C, Falzone TL, et al. Axonopathy and transport deficits early in the pathogenesis of Alzheimer's disease. Science 2005;307:1282-8.

10. Wang DM, Li SQ, Zhu XY, et al. Protective effects of hesperidin against amyloid- $\beta(\mathrm{A} \beta)$ induced neurotoxicity through the voltage dependent anion channel 1 (VDAC1)mediated mitochondrial apoptotic pathway in PC12 cells. Neurochem Res 2013;38:1034-44.

11. Di Re J, Wadsworth PA, Laezza F. Intracellular Fibroblast Growth Factor 14: Emerging Risk Factor for Brain Disorders. Front Cell Neurosci 2017;11:103.

12. Tiwari S, Atluri V, Kaushik A, et al. Alzheimer's disease: pathogenesis, diagnostics, and therapeutics. Int J Nanomedicine 2019;14:5541-54.

13. Chen JX, Yan SS. Role of mitochondrial amyloid-beta in Alzheimer's disease. J Alzheimers Dis 2010;20 Suppl 2:S569-78.

14. Kam TI, Gwon Y, Jung YK. Amyloid beta receptors responsible for neurotoxicity and cellular defects in Alzheimer's disease. Cell Mol Life Sci 2014;71:4803-13.

15. Liu R, Gao M, Qiang GF, et al. The anti-amnesic effects of luteolin against amyloid beta(25-35) peptide-induced toxicity in mice involve the protection of neurovascular unit. Neuroscience 2009;162:1232-43.

16. Piarroux J, Riant F, Humbertclaude V, et al. FGF14-related episodic ataxia: delineating the phenotype of Episodic Ataxia type 9. Ann Clin Transl Neurol 2020;7:565-72.

17. Alshammari MA, Alshammari TK, Nenov MN, et al. Fibroblast Growth Factor 14 Modulates the Neurogenesis of Granule Neurons in the Adult Dentate Gyrus. Mol Neurobiol 2016;53:7254-70.

18. Bhatwa A, Wang W, Hassan YI, et al. Challenges Associated With the Formation of Recombinant Protein Inclusion Bodies in Escherichia coli and Strategies to Address Them for Industrial Applications. Front Bioeng Biotechnol 2021;9:630551.

19. Singh A, Upadhyay V, Upadhyay AK, et al. Protein recovery from inclusion bodies of Escherichia coli using mild solubilization process. Microb Cell Fact 2015;14:41.

20. Xu N, Wang BH, Zhou Q, et al. Expression of HalohFGF18 and study of its effect on differentiation of ATDC5 cells. Protein Expr Purif 2019;155:8-14.

21. Wang S, Lin H, Zhao T, et al. Expression and purification of an FGF9 fusion protein in E. coli, and the effects of the FGF9 subfamily on human hepatocellular carcinoma cell proliferation and migration. Appl Microbiol Biotechnol 2017;101:7823-35.

22. Crews L, Masliah E. Molecular mechanisms of neurodegeneration in Alzheimer's disease. Hum Mol Genet 2010;19:R12-20.

23. Mecocci P, Boccardi V, Cecchetti R, et al. A Long Journey into Aging, Brain Aging, and Alzheimer's Disease Following the Oxidative Stress Tracks. J Alzheimers Dis 2018;62:1319-35.

24. Misrani A, Tabassum S, Yang L. Mitochondrial Dysfunction and Oxidative Stress in Alzheimer's Disease. Front Aging Neurosci 2021;13:617588.

25. Albert-Gascó H, Ros-Bernal F, Castillo-Gómez E, et al. MAP/ERK Signaling in Developing Cognitive and Emotional Function and Its Effect on Pathological and Neurodegenerative Processes. Int J Mol Sci 2020;21:4471.

26. Lee JK, Kim NJ. Recent Advances in the Inhibition of p38 MAPK as a Potential Strategy for the Treatment of Alzheimer's Disease. Molecules 2017;22:1287.

27. Zhu X, Raina AK, Rottkamp CA, et al. Activation and redistribution of c-jun $\mathrm{N}$-terminal kinase/stress activated protein kinase in degenerating neurons in Alzheimer's disease. J Neurochem 2001;76:435-41.

28. Killick R, Ribe EM, Al-Shawi R, et al. Clusterin regulates $\beta$-amyloid toxicity via Dickkopf-1-driven induction of the wnt-PCP-JNK pathway. Mol Psychiatry 2014;19:88-98. 
29. Musi CA, Agrò G, Santarella F, et al. JNK3 as Therapeutic Target and Biomarker in Neurodegenerative and Neurodevelopmental Brain Diseases. Cells 2020;9:2190.

30. Kelleher RJ 3rd, Govindarajan A, Tonegawa S. Translational regulatory mechanisms in persistent forms of synaptic plasticity. Neuron 2004;44:59-73.

Cite this article as: Wang L, Jing R, Wang X, Wang B, Guo K, Zhao J, Gao S, Xu N, Xuan X. A method for the expression of fibroblast growth factor 14 and assessment of its neuroprotective effect in an Alzheimer's disease model. Ann Transl Med 2021;9(12):994. doi: 10.21037/atm-21-2492
31. Giovannini MG. The role of the extracellular signalregulated kinase pathway in memory encoding. Rev Neurosci 2006;17:619-34.

(English Language Editor: J. Reynolds) 